\title{
Impacto da incontinência urinária na qualidade de vida de mulheres: revisão integrativa da literatura
}

\author{
Impact of urinary incontinence on women's quality of life: an integrative literature review \\ Impacto de la incontinencia urinaria en la calidad de vida de las mujeres: una revisión bibliográfica \\ integradora
}

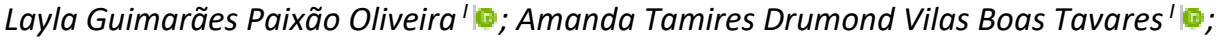

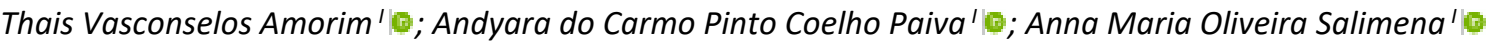

'Universidade Federal de Juiz de Fora, Juiz de Fora, MG, Brasil

\begin{abstract}
RESUMO
Objetivo: verificar o impacto da incontinência urinária na qualidade de vida das mulheres. Método: revisão integrativa realizada em fontes eletrônicas da CAPES, LILACS e PubMed. Utilizou-se como critério de inclusão, os artigos disponíveis eletronicamente na íntegra e que dissertaram sobre a temática, e de exclusão os que apresentaram duplicidade na busca. Foram selecionados doze artigos. Resultados: os estudos analisados foram publicados no período de 2015 a 2019, sendo nove estudos do tipo transversal. Identificaram-se quatro categorias temáticas: percepção das mulheres sobre a IU; impacto da IU na QV de mulheres; tipo de IU de maior influência sobre a QV; e importância da avaliação da QV de mulheres incontinentes. Conclusão: evidenciouse o impacto negativo da IU na QV de mulheres, devido a limitações físicas, sexuais, ocupacionais e sociais, e sentimentos como vergonha, falta de controle, mal-estar, insegurança, sofrimento e culpa.
\end{abstract}

Descritores: Mulheres; Saúde da Mulher; Incontinência Urinária; Qualidade de Vida.

\begin{abstract}
Objective: to verify the impact of urinary incontinence on women's quality of life. Method: integrative review conducted in CAPES, LILACS and PubMed electronic sources. For inclusion, articles had to be available in full electronically and address the subject; search duplicates were excluded. Twelve articles were selected. Results: these studies, nine cross-sectional, were published from 2015 to 2019. Four thematic categories were identified: women's perception of UI; impact of UI on women's QOL; UI type with greatest impact on QOL; and importance of assessing incontinent women's QOL. Conclusion: UI had adverse impact on QOL of women, because of physical, sexual, occupational, and social limitations, as well as feelings of shame, lack of control, discomfort, insecurity, suffering and guilt.
\end{abstract}

Descriptors: Women's; Women Health; Urinary Incontinence; Quality of Life.

\section{RESUMEN}

Objetivo: verificar el impacto de la incontinencia urinaria en la calidad de vida de las mujeres. Método: revisión integradora realizada en fuentes electrónicas CAPES, LILACS y PubMed. Para su inclusión, los artículos debían estar disponibles en su totalidad en formato electrónico y abordar el tema; Se excluyeron los duplicados de búsqueda. Se seleccionaron doce artículos. Resultados: estos estudios, nueve transversales, se publicaron entre 2015 y 2019. Se identificaron cuatro categorías temáticas: percepción de las mujeres sobre la IU; impacto de la IU en la calidad de vida de las mujeres; Tipo de IU con mayor impacto en la calidad de vida; e importancia de evaluar la calidad de vida de las mujeres con incontinencia. Conclusión: la IU tuvo un impacto adverso en la calidad de vida de las mujeres, debido a limitaciones físicas, sexuales, ocupacionales y sociales, así como sentimientos de vergüenza, descontrol, malestar, inseguridad, sufrimiento y culpa.

Descriptores: Mujeres; Salud de la Mujer Incontinencia Urinaria; Calidad de Vida.

\section{INTRODUÇÃO}

Incontinência Urinária (IU) é denominada pela Sociedade Internacional de Continência (ICS) como qualquer perda involuntária de urina, e considerada como uma doença na Classificação Internacional de Doenças (CID 10/OMS) ${ }^{1,2}$. Apontada como um problema de saúde pública, essa patologia acomete mais de 200 milhões de pessoas no mundo, e apresenta uma elevada taxa de prevalência em mulheres, que aumenta com o avanço da idade ${ }^{3}$.

Vista como uma disfunção de alta incidência na sociedade moderna, cerca de 20 a $50 \%$ das mulheres adultas podem apresentar IU em alguma fase da vida, sendo que, para a cada um homem duas mulheres são incontinentes ${ }^{4,5}$. No Brasil, aproximadamente de 30 a $43 \%$ das mulheres sofrem com a perda involuntária de urina em algum momento de sua vida, porém esses valores podem estar abaixo da realidade, pelo fato de tal patologia permanecer subdiagnosticada e subtratada ${ }^{3,4}$. 
A IU pode ser classificada de acordo com a sua etiologia e sintomatologia, sendo os tipos mais frequentes: a incontinência urinária de esforço (IUE), caracterizada como a perda de urina devido ao esforço, que causa o aumento da pressão intra-abdominal, como tosse, espirro e atividade física; a incontinência urinária de urgência (IUU) que é a perda de urina associada à forte sensação de urgência para urinar; e a incontinência urinária mista (IUM), quando ocorre a perda de urina relacionada à urgência e aos esforços ${ }^{6,7}$.

Apesar de possuir uma origem multifatorial a IU feminina apresenta alguns fatores de riscos específicos como: gravidez, tipo de parto, menopausa, histerectomia, episiotomia vaginal e índice de massa corporal ${ }^{8}$. 0 escape involuntário de urina é uma condição capaz de provocar limitações físicas, sociais, ocupacionais, domésticas e sexuais, comprometer os aspectos biopsicossociais e apresentar grande impacto na qualidade de vida (QV) das mulheres ${ }^{9}$.

O termo QV apresenta duas conceituações na área da saúde de acordo com a Organização Mundial de Saúde. E, em seu conceito mais genérico, é definida como "a percepção do indivíduo sobre a sua posição na vida, no contexto da cultura e dos sistemas de valores nos quais ele vive, e em relação a seus objetivos, expectativas, padrões e preocupações"; e em seu conceito mais específico, a qualidade de vida relacionada à saúde (QVRS) é associada com os "aspectos da vida de uma pessoa que apresenta alterações em seu estado de saúde, significativos para impactar a sua qualidade de vida", apresentando, então, uma relação direta com enfermidades e intervenções em saúde ${ }^{10}$.

Avaliar o impacto da IU perante a QVRS de mulheres incontinentes torna-se uma estratégia significativa para traçar o perfil dessa patologia não apenas pelo olhar clínico, mas também pela ótica da paciente. Logo, possibilita o desenvolvimento de um processo terapêutico eficiente, em busca da recuperação da continência e da QV das pacientes ${ }^{11}$. Para realizar essa avaliação, pode ser utilizado questionários específicos, válidos e fidedignos, que mensuram a gravidade e o impacto dos sintomas da IU sobre a QV, por exemplo: o International Consultation on Incontinence Questionnaire-Short Form (ICIQ-SF), o Incontinence-Specific Quality of Life Instrument (I-QOL) e o King's Health Questionnaire (KHQ) $)^{12,13}$

O questionário ICIQ-SF é considerado simples, breve e auto-administrável, por meio do qual avaliam-se, por meio de quatro perguntas, a frequência, a gravidade e o impacto da IU na QV de homens e mulheres. Apresenta também um conjunto de oito itens de autodiagnóstico, relacionados às causas ou situações vivenciadas pelos pacientes. Portanto, é um instrumento que pode ser utilizado em pesquisas científicas e na prática clínica, servindo para rastrear e qualificar a perda urinária ${ }^{14}$.

O I-QOL vai avaliar o impacto da IUE na QV de pacientes em ambos os sexos, por meio de vinte e duas perguntas que são divididas em três domínios (limitação de comportamento, impacto psicológico e o embaraço social). Como o ICIQ-SF, esse questionário também pode ser utilizado tanto em pesquisas científicas quanto na prática clínica diária, podendo ser conduzido em entrevistas pessoais ou por telefone ${ }^{15}$.

O KHQ validado no Brasil em 2003, é o questionário mais utilizado em estudos sobre QV e IU. Seu objetivo é avaliar a percepção do impacto da IU na QV de mulheres, por meio de vinte e uma questões e oito domínios (percepção geral da saúde, impacto da IU, limitações das atividades diárias, limitações físicas, limitações sociais, relacionamentos pessoais, emoções, sono e energia). Os escores obtidos em cada domínio vai determinar o impacto da IU sobre a QV, ou seja, maiores escores indicam um maior impacto ${ }^{13}$.

Diante do exposto e da relevância do tema, o estudo teve como pergunta norteadora: Qual é o impacto da incontinência urinária sobre a qualidade de vida das mulheres incontinentes? Para responder essa indagação, objetivouse verificar o impacto da incontinência urinária na qualidade de vida das mulheres, por meio de uma revisão integrativa de literatura, fornecendo conhecimentos fundamentais dessa temática à luz da literatura publicada.

\section{MÉTODO}

Trata-se de uma revisão integrativa da literatura, método científico que busca avaliar e sintetizar resultados de pesquisas anteriores, apresentando conclusões do corpus literário sobre um determinado fenômeno de forma sistemática, ordenada e abrangente ${ }^{16}$.

Para a realização desta, foram utilizados os seguintes passos do método: identificação do tema e seleção da questão de pesquisa; estabelecimento dos critérios de inclusão e exclusão; definição das informações a serem extraídas dos estudos selecionados; avaliação dos estudos incluídos na revisão integrativa; interpretação dos resultados; e apresentação da revisão do conhecimento ${ }^{17}$.

A coleta de dados ocorreu no período de fevereiro a março de 2020, por meio do portal de Periódicos da CAPES, LILACS e PubMed, recorrendo à combinação das seguintes palavras-chave reconhecidas pelo Descritores em Ciências de Saúde (DeCS): Incontinência Urinária (Urinary Incontinence); Qualidade de Vida (Quality of Life); e Saúde da Mulher (Women's Health). Esses termos foram buscados nas bases de dados utilizando o operador lógico booleano AND, e cruzados como descritores, palavras do título e resumo. 
Para o refinamento da pesquisa e definição da amostra, foram considerados os seguintes critérios de inclusão: artigos completos indexados no portal de Periódicos da CAPES, LILACS e PubMed, publicados no período de 2015 até março de 2020, disponível eletronicamente na íntegra em português, inglês, ou espanhol, que abordassem de forma direta o objeto de pesquisa e respondessem à pergunta norteadora. Foram excluídos os artigos que apresentaram duplicidade na busca, ou que não contemplaram os critérios de inclusão definidos.

Inicialmente, para a realização desta revisão, foram encontrados 86 artigos indexados no portal de periódicos da CAPES, 150 artigos na PubMed e dois artigos na LILACS, totalizando 238 artigos. Destes, após o processo de busca com a aplicação dos critérios de inclusão e exclusão, foi possível selecionar 86 artigos científicos, para realizar uma pré-leitura exploratória dos títulos e resumos. Por meio desta elegibilidade, foram escolhidos 21 artigos, os quais foram lidos integralmente, verificando se atendiam os critérios de inclusão e se respondiam à pergunta norteadora desta revisão.

Mediante avaliação e caracterização quanto a título, autoria, periódico, ano de publicação, método e principais resultados, a fim de organizar e tabular as informações dos estudos selecionados, excluíram-se nove artigos, pois não apresentaram uma abordagem direta do tema e não responderam à pergunta norteadora. Portanto, conforme disposto no Fluxograma da Figura 1, foram selecionadas 12 publicações para realizar a análise, por meio de uma leitura pertinente e crítica, a fim de extrair informações para o alcance do objetivo deste estudo.

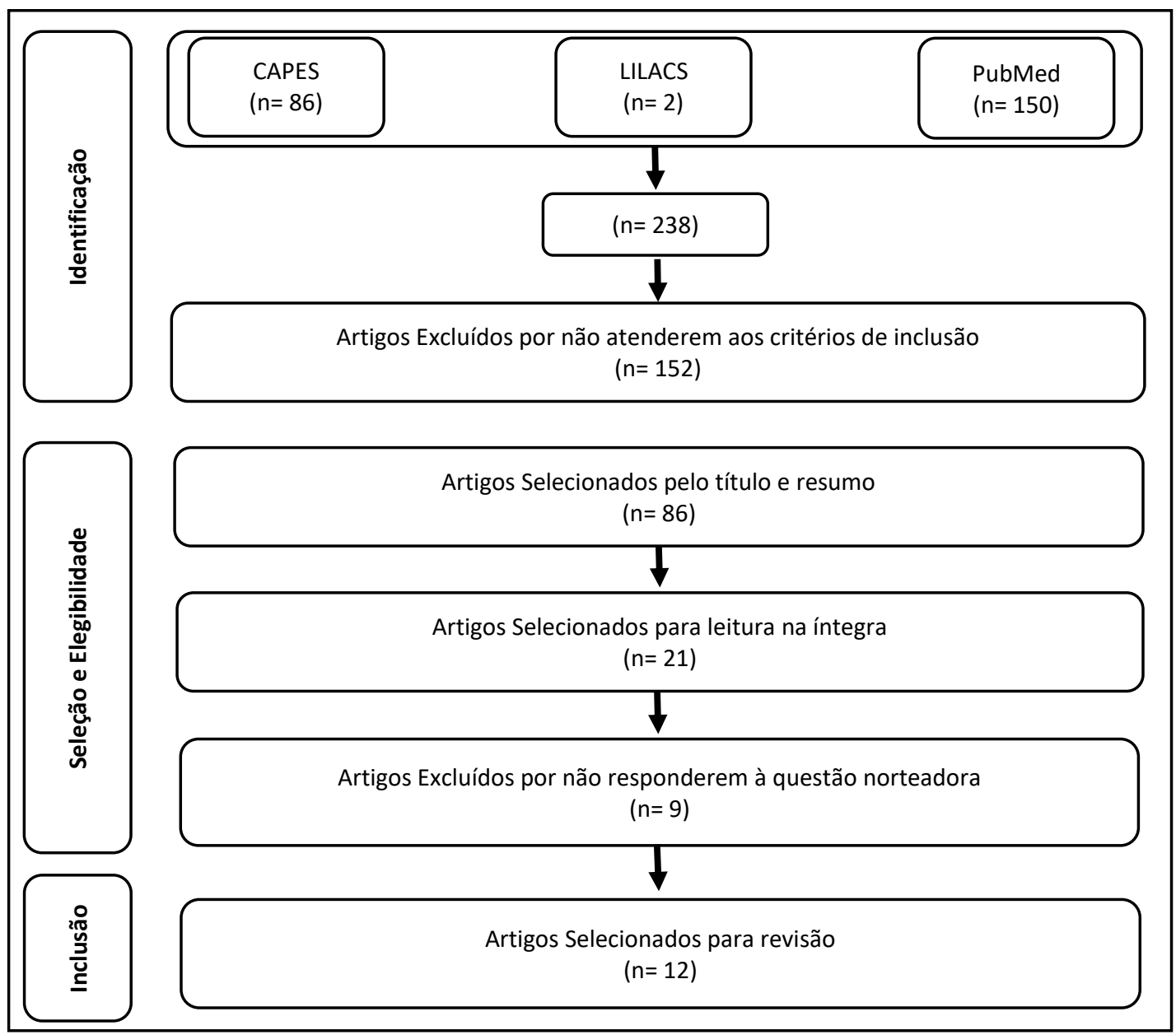

FIGURA 1: fluxograma explicativo sobre a etapa de busca de artigos no portal de Periódicos da CAPES, LILACS e PubMed. Juiz de Fora, MG, Brasil, 2020.

Após a realização da análise, agruparam-se os conteúdos semelhantes em que emergiram as seguintes temáticas: a percepção das mulheres sobre a IU; o impacto da IU na QV das mulheres; o tipo de IU de maior influência sobre a QV; e a importância da avaliação da QV de mulheres incontinentes. 


\section{RESULTADOS E DISCUSSÃO}

Os 12 artigos científicos selecionados para compor este estudo foram categorizados e explicitados na Figura 2.

\begin{tabular}{|c|c|c|c|}
\hline Título/Ano & $\begin{array}{c}\text { Periódico/ } \\
\text { Base de dados }\end{array}$ & Método & Principais Resultados \\
\hline $\begin{array}{l}\text { Perfil e qualidade de vida de mulheres } \\
\text { em reabilitação do assoalho pélvico, } \\
2018^{12}\end{array}$ & $\begin{array}{l}\text { REBEn } \\
\text { CAPES }\end{array}$ & $\begin{array}{l}\text { Estudo } \\
\text { Transversal }\end{array}$ & $\begin{array}{l}\text { A mudança na atividade sexual e alguns tipos de } \\
\text { IU afetaram a QVRS. }\end{array}$ \\
\hline $\begin{array}{l}\text { Living with UI: potential risks of women's } \\
\text { health? A qualitative study on the } \\
\text { perspectives of female patients seeking } \\
\text { care for the first time in a specialized } \\
\text { center, } 2019^{18}\end{array}$ & $\begin{array}{l}\text { Int. J. Environ. Res. } \\
\text { Public Health } \\
\text { PubMed }\end{array}$ & $\begin{array}{l}\text { Estudo } \\
\text { Exploratório } \\
\text { Qualitativo }\end{array}$ & $\begin{array}{l}\text { A IU foi definida como algo desagradável, } \\
\text { estressante e traumático, que remete a } \\
\text { sentimentos de vergonha, insegurança, } \\
\text { sofrimento e culpa. Logo, determinada como } \\
\text { uma experiência negativa. }\end{array}$ \\
\hline $\begin{array}{l}\text { O impacto da IU sobre a qualidade de } \\
\text { vida e sua relação com a sintomatologia } \\
\text { depressiva e ansiedade em mulheres, } \\
2019^{19}\end{array}$ & $\begin{array}{l}\text { Rev. Salud Pública } \\
\text { CAPES }\end{array}$ & $\begin{array}{l}\text { Estudo } \\
\text { Transversal }\end{array}$ & $\begin{array}{l}\text { Verificou-se elevada prevalência de depressão e } \\
\text { ansiedade, associada ao pior impacto na QV das } \\
\text { mulheres incontinentes. }\end{array}$ \\
\hline $\begin{array}{l}\text { UI among urban and rural community } \\
\text { dwelling older women: prevalence, risk } \\
\text { factors and quality of life, } 2019^{20}\end{array}$ & $\begin{array}{l}\text { BMC Public Health } \\
\text { PubMed }\end{array}$ & $\begin{array}{l}\text { Análise de } \\
\text { dados }\end{array}$ & $\begin{array}{l}\text { As mulheres das áreas rurais que apresentavam } \\
\text { IU, experimentaram maiores limitações físicas, } \\
\text { sociais, emocionais e distúrbios do sono em } \\
\text { comparação aos seus pares urbanos. }\end{array}$ \\
\hline $\begin{array}{l}\text { Investigação da qualidade de vida de } \\
\text { mulheres com IU, } 2018^{21}\end{array}$ & $\begin{array}{l}\text { Arq. Ciênc. Saúde } \\
\text { UNIPAR } \\
\text { LILACS }\end{array}$ & $\begin{array}{l}\text { Estudo } \\
\text { Transversal }\end{array}$ & $\begin{array}{l}\text { Constatou-se por meio dos instrumentos ICQ-SF } \\
\text { e KHQ, o impacto negativo da IU sobre a QV das } \\
\text { mulheres. }\end{array}$ \\
\hline $\begin{array}{l}\text { Female urinary incontinence: quality of } \\
\text { life comparison on reproductive age and } \\
\text { postmenopausal period, } 2016^{22}\end{array}$ & $\begin{array}{l}\text { Fisioter Mov. } \\
\text { CAPES }\end{array}$ & $\begin{array}{l}\text { Estudo } \\
\text { Descritivo- } \\
\text { Retrospectivo }\end{array}$ & $\begin{array}{l}\text { Na amostra estudada a IU afetou negativamente } \\
\text { a QV, porém houve um impacto } \\
\text { significativamente maior da patologia em } \\
\text { mulheres no período pós-menopausa. }\end{array}$ \\
\hline $\begin{array}{l}\text { O impacto da IU feminina na qualidade } \\
\text { de vida, } 2016^{24}\end{array}$ & $\begin{array}{l}\text { Educação e Ciência } \\
\text { para a Cidadania } \\
\text { Global } \\
\text { CAPES }\end{array}$ & $\begin{array}{l}\text { Estudo } \\
\text { Transversal }\end{array}$ & $\begin{array}{l}\text { Os resultados mostraram que a QV de mulheres } \\
\text { com IU é afetada de forma negativa, provocando } \\
\text { sentimentos de baixa autoestima, interferindo nas } \\
\text { atividades diárias e restringindo o contato social. }\end{array}$ \\
\hline $\begin{array}{l}\text { Impacto do tipo de IU sobre a qualidade } \\
\text { de vida de usuárias do SUS no Sudeste do } \\
\text { Brasil, } 2015^{25}\end{array}$ & $\begin{array}{l}\text { Rev. Bras. Ginecol } \\
\text { Obstet. } \\
\text { CAPES }\end{array}$ & $\begin{array}{l}\text { Estudo } \\
\text { Transversal }\end{array}$ & $\begin{array}{l}\text { Foi possível observar neste estudo, que mulheres } \\
\text { com IUM, apresentaram piores escores no } \\
\text { questionário de avaliação KHQ e comprometeu } \\
\text { de forma moderada/grave a QV. }\end{array}$ \\
\hline $\begin{array}{l}\text { Quality of life of women with urinary } \\
\text { incontinence in rehabilitation treatment, } \\
2016^{26}\end{array}$ & $\begin{array}{l}\text { Journal of Health } \\
\text { Psychology } \\
\text { PubMed }\end{array}$ & $\begin{array}{l}\text { Estudo } \\
\text { Transversal }\end{array}$ & $\begin{array}{l}\text { Identificou-se que a satisfação sexual, a } \\
\text { gravidade, o sofrimento e o impacto da IU } \\
\text { predizem a QV. }\end{array}$ \\
\hline $\begin{array}{l}\text { Impacto dos tipos de IU na qualidade de } \\
\text { vida de mulheres, } 2017^{27}\end{array}$ & $\begin{array}{l}\text { Rev. Esc. Enferm. } \\
\text { USP } \\
\text { CAPES }\end{array}$ & $\begin{array}{l}\text { Estudo } \\
\text { Transversal }\end{array}$ & $\begin{array}{l}\text { Todos os tipos de IU interferem tanto na QV } \\
\text { geral, quanto na específica, porém as mulheres } \\
\text { com IUM são as mais afetadas. }\end{array}$ \\
\hline $\begin{array}{l}\text { Qualidade de vida em mulheres com IU, } \\
2015^{28}\end{array}$ & $\begin{array}{l}\text { Revista de } \\
\text { Enfermagem } \\
\text { Referência } \\
\text { CAPES }\end{array}$ & $\begin{array}{l}\text { Estudo } \\
\text { Transversal }\end{array}$ & $\begin{array}{l}\text { A IU influencia moderadamente a QV da mulher, } \\
\text { sendo a IUE a que causa um pior impacto. }\end{array}$ \\
\hline $\begin{array}{l}\text { Urinary incontinence and quality of life of } \\
\text { women living in nursing homes in the } \\
\text { Mediterranean region of Turkey, } 2017^{29}\end{array}$ & $\begin{array}{l}\text { PSYCHOGERIATRICS } \\
\text { PubMed }\end{array}$ & $\begin{array}{l}\text { Estudo } \\
\text { Transversal }\end{array}$ & $\begin{array}{l}\text { Contatou-se que a QV das mulheres que não } \\
\text { consideravam a IU como um problema de saúde, } \\
\text { foi significativamente maior do que daquelas que } \\
\text { consideravam. A IUM foi o subtipo mais comum } \\
\text { e identificada como a que mais afeta a QV. }\end{array}$ \\
\hline
\end{tabular}

FIGURA 2: Distribuição dos artigos selecionados segundo título, referência, autor, periódico/ano, método e principais resultados. Juiz de Fora, MG, Brasil, 2020.

Dos estudos selecionados, seis destes foram publicados em 2016 e 2019, sendo três em cada ano, e nos anos de 2015, 2017 e 2018, foram dois artigos em cada. No que se refere ao idioma, cinco publicações foram em inglês, e sete, em português. Destaca-se que todos os artigos são originais, sendo nove estudos do tipo transversal, e publicados em periódicos diferentes relacionados com a área da saúde. 
Todos os estudos buscaram como parte do objetivo analisar, avaliar ou investigar o impacto/influência da IU sobre a QV de mulheres. Como instrumento de coleta de informações para a mensuração dessa QV, nove utilizaram o King's Health Questionnaire (KHQ), dois escolheram questionários mais específicos de mensuração (PRAFAB-questionnaire e Incontinence-Specific Quality of Life Instrument), e um usou como método a entrevista semiestruturada. Quatro dos artigos que aplicaram o KHQ como questionário, usaram também outros questionários como o ICIQ-SF e o I-QOL, em busca de comparar dados e trazer informações mais precisas a respeito do impacto da IU sobre a QV de mulheres incontinentes.

A amostra analisada nessa revisão abordou alguns aspectos semelhantes como: o impacto da IU sobre a QV de mulheres; a percepção da QV de mulheres com IU; a influência de tipos específicos de IU sobre a QV; a influência de variáveis como sociodemográficas, obstétricas, ginecológicas e clínicas na IU e consequentemente na QV; e a importância da avaliação da QV de mulheres incontinentes. Desses, surgiram as categorias temáticas Percepção das mulheres sobre a IU, Impacto da IU na QV de mulheres, Tipo de IU de maior influência sobre a QV e Importância da avaliação da QV de mulheres incontinentes, que apresentam a síntese do conhecimento contemplado na literatura.

\section{Percepção das mulheres sobre a IU}

Os sintomas provocados pela IU podem afetar adversamente a rotina de uma mulher. As perspectivas e as narrativas sobre o impacto dessa patologia são imprescindíveis para sua compreensão, principalmente sob o olhar de pacientes incontinentes ${ }^{18}$.

As mulheres percebem a IU como uma experiência negativa, desagradável e estressante, que causa sentimentos de vergonha, falta de controle, mal-estar, insegurança, sofrimento e culpa ${ }^{18-20}$. Estudiosos relatam que as mulheres ao conviver com a perda involuntária de urina, sentem-se sujas e impuras, descrevendo essa situação como defeito ou falha delas, a ponto de experienciar culpa por serem daquela forma ${ }^{18}$.

Esses sentimentos podem acarretar fatores como: isolamento e exclusão social, baixa autoestima feminina, depressão, ansiedade e atraso na busca de tratamento, influenciando diretamente na QV dessas mulheres, e forçandoas passar por mudanças de hábitos diários. A falta de informação sobre a patologia, crenças falsas e mitos podem potencializar a percepção negativa ${ }^{19,20}$.

\section{Impacto da IU na QV de mulheres}

A IU influencia a QV de mulheres em várias dimensões, podendo ocasionar alterações psicoemocionais e limitações como físicas, sociais, sexuais e de atividade diárias ${ }^{21}$. Pesquisadores relatam que esse impacto está relacionado a percepção individual de gravidade, tipo e quantidade de perda de urina e o contexto cultural em que essas estão inseridas ${ }^{22}$.

As limitações físicas e sociais estão relacionadas ao medo ou vergonha de perder urina em público, o que leva as mulheres a deixarem de sair de casa, de ir a festas e à igreja, de fazer compras, de realizar longas viagens e praticar atividades físicas, por receio de aparentar estarem com a roupa molhada, cheirando urina ou de não encontrar um banheiro por perto. Muitas dessas mulheres só saem de casa ao saber que haverá facilidade para utilizar o banheiro quando houver necessidade ${ }^{23,24}$.

As limitações na atividade sexual estão associadas ao incômodo de sentir vontade de urinar ou da perda urinária durante a relação, podendo ocasionar a perda do prazer e do desejo sexual. Diante disso, as mulheres diminuem e até evitam a prática desta atividade ${ }^{23,25}$.

Quanto às atividades diárias, destacam-se limitações como realizar serviços domésticos que requeiram esforço físico ou carregar peso, ações que influenciam na perda de urina e provocam maiores idas ao banheiro ou o escape na roupa. O aumento das idas aos banheiros durante o dia a dia provoca limitações também nas atividades ocupacionais, o que interfere no desempenho profissional da mulher incontinente, por necessitar interromper o trabalho diversas vezes. Diante desse fator, muitas sentem-se tão constrangidas que pedem demissão do trabalho ${ }^{23,24}$. Essas restrições podem gerar frustações e demonstram a influência e o impacto da IU na QV da mulher incontinente ${ }^{23}$.

Os estudos analisados ${ }^{12,18-22,24-29}$, de forma unânime, constataram que a IU impactou negativamente a QV de mulheres, de modo grave/muito grave. Os efeitos negativos na percepção geral da saúde, nas limitações que são ocasionadas e no impacto da IU interferem na QV, porém o principal indicador dessa ser diminuída é o nível de gravidade da patologia 21,26 .

Algumas variáveis quantitativas e qualitativas, como sexo, idade e condições clínicas e sociodemográficas, podem potencializar esse impacto negativo da IU sobre a QV. Tal aspecto foi identificado em estudo ${ }^{20}$, em que mulheres idosas com IU que viviam em meio urbano apresentavam uma maior prevalência, enquanto mulheres na mesma condição, mas que viviam em meio rural tinham um maior impacto negativo em sua QV. Algumas sintomatologias também podem 
intensificar essa baixa QV como foi constatado em outro estudo ${ }^{19}$, no qual mulheres com IU caracterizadas como depressivas e ansiosas apresentavam pior QV, comparadas a mulheres apenas incontinentes.

Todos os domínios da QV avaliados pelo questionário KHQ podem ser comprometidos pela IU, principalmente a percepção geral da saúde, as limitações das atividades diárias, as relações pessoais e as emoções. Esses apresentaram maiores médias de escores nos estudos, e evidenciam o impacto negativo dessa patologia na QV de mulheres ${ }^{12,24,26}$.

\section{Tipo de IU de maior influência sobre a QV}

Os domínios presentes nos questionários ICIQ-SF, I-QOL e no KHQ podem apresentar variação quanto ao seu comprometimento conforme o subtipo de IU, sendo a incontinência urinária mista (IUM) responsável por maiores escores e, consequentemente, menor índice de QV. Os estudos ${ }^{19,25,27}$ identificaram que esse subtipo de incontinência tem uma maior influência sobre as restrições das relações pessoais e das atividades diárias e um maior impacto emocional. Neste contexto, pesquisadore ${ }^{25}$ evidenciaram grande impacto também na atividade sexual, pois perder urina durante a relação sexual pode causar ansiedade, diminuição da libido e conflitos no relacionamento, potencializando o efeito negativo na QV.

A IUM apresenta pior impacto na QV devido a sua maior gravidade clínica e, diferente de outros subtipos, não tem situações previsíveis de perda de urina, como a incontinência urinária de esforço (IUE), sendo difícil encontrar modalidades para reduzir seu impacto negativo no cotidiano ${ }^{19,27}$.

\section{Importância da avaliação da QV de mulheres incontinentes}

Diante de todos os impactos e circunstâncias que a IU pode ocasionar na vida de uma mulher, os estudos ${ }^{21,22,26}$ comprovam a necessidade de considerar a análise da QV, por meio de questionários específicos, durante a avaliação da rotina de uma incontinente. Esses questionários devem ser direcionados para a IU e seu impacto, podendo ser utilizado mais de um tipo, possibilitando realizar uma análise completa, e identificar a gravidade e a influência dessa patologia sobre as pacientes ${ }^{21}$.

Desta forma, a investigação da QV deve ser considerada como um parâmetro central na avalição da IU, no qual as ações dos profissionais de saúde durante o planejamento e orientação do tratamento vão se centrar, revelando-se essencial para o processo de recuperação ${ }^{12,22,27}$. Logo, esses profissionais devem priorizar desenvolver uma assistência focada na promoção da saúde e em processos terapêuticos, que busquem principalmente diminuir os aspectos negativos da IU na QV das mulheres ${ }^{12,22,27-29}$.

\section{CONCLUSÃO}

A IU é experenciada pelas mulheres como algo nocivo, que ocasiona um grande incômodo físico e emocional, além de influenciar de maneira significativa os hábitos diários. A falta de informação, o subtipo de incontinência, a gravidade dos sintomas, as características sociodemográficas e uroginecológicas, podem potencializar negativamente essa vivência.

Evidencia-se, então, o impacto negativo da IU na QV de mulheres, devido a alterações psicoemocionais, limitações físicas, sexuais, ocupacionais e sociais, e sentimentos como vergonha, falta de controle, mal-estar, insegurança, sofrimento e culpa. Esse impacto na QV pode variar de acordo com o subtipo de IU, sendo a IUM caracterizada como a que mais compromete a QV, principalmente as atividades diárias, as emoções e as relações pessoais.

Constatou-se que a compreensão e a avaliação da QV de mulheres incontinentes devem se apresentar como um parâmetro central da prática clínica, para delimitar uma terapia completa que visa às necessidades da paciente e busca reduzir os aspectos negativos da IU. É necessário e importante desmistificar a incontinência, disponibilizando informações para essas mulheres sobre a patologia, a importância da ajuda profissional e as estratégias terapêuticas.

Consideram-se, como limitações do estudo, a amostra e a busca nas fontes eletrônicas selecionadas, procedimento que restringiu a busca em três bases de dados, e, assim, foram incluídos apenas os artigos disponíveis on-line na íntegra, sendo que, desses, apenas um estudo utilizou o método qualitativo.

Por conseguinte, após o conhecimento da literatura sobre a temática, recomenda-se que seja realizada ampliação na busca e produção de novos estudos qualitativos, sobre a compreensão do impacto da IU na vida de mulheres, a fim de contribuir para o esclarecimento sobre a vivência deste fenômeno e intervenções específicas para essa população.

\section{REFERÊNCIAS}

1. ICS. International Continence Society [site de Internet]. Current definitions. [cited 2020 may 14] Available from: https://www.ics.org/terminology/113.

2. CID-10. Classificação Estatística Internacional de Doenças e Problemas Relacionados à Saúde [site de Internet]. CID10Classificação Internacional de Doenças: incontinência urinária não especificada. [cited 2020 may 20]. Available from: https://www.cid10.com.br/buscadescr?query=R32+++Incontinencia+urinaria+NE. 
3. Norton P, Brubaker L. Urinary incontinence in women. The Lancet [Internet], 2006 [cited 2020 may 14]; 367(9504):57-67. DOI: https://doi.org/10.1016/S0140-6736(06)67925-7.

4. Figueiredo EM, Lara JO, Cruz MC, Quintão DMG, Monteiro MVC. Sociodemographic and clinical profi le of female users of public Urogynecological Physical Therapy Services. Rev. bras. Fisioter [Internet], 2008 [cited 2020 may 14]; 12(2):136-42. DOI: https://doi.org/10.1590/S1413-35552008000200010.

5. Sacomori C, Boer L, Sperandio F, Cardoso FL. Prevalence of variables associated with urinary incontinence in the third trimester of pregnancy. Rev. Bras. Saúde Mater. Infant. [Internet], 2013 [cited 2020 may 14]; 13(3):215-21. DOI: https://doi.org/10.1590/S1519-38292013000300003.

6. Borba AMCde, Lelis MAdosS, Brêtas ANP. The meaning of urinary incontinence and be incontinent - women opinion. Texto contexto - enferm. [Internet], 2008 [cited 2020 may 14]; 17(3); 527-35. DOI: https://doi.org/10.1590/S010407072008000300014

7. Higa R, Lopes MHBdeM, Reis MJ. Risk factors for urinary incontinence in women. Rev. Esc. Enferm. [Internet], 2008 [cited 2020 may 14]; 42(1):187-92. DOI: https://doi.org/10.1590/S0080-62342008000100025.

8. Demircan N, Özmen Ü, Köktürk F, Kücük H, Ata S, Harma M, Arıkan ii. What are the probable predictors of urinary incontinence during pregnancy?. PeerJ [Internet], 2016 [cited 2020 may 14]; 1-19. DOI: https://doi.org/10.7717/peerj.2283.

9. KILIC, M. Incidence and risk factors of urinary incontinence in women visiting family health centers. Springer Plus, 2016 [cited 2020 may 14]; 5(1331):1-9. DOI: https://dx.doi.org/10.1186\%2Fs40064-016-2965-z.

10. Seidl EMF, Zannon CMLdaC. Quality of life and health: conceptual and methodological issues. Cad. Saúde Pública [Internet], 2004 [cited 2020 may 14]; 20(2):580-8. DOI: http://dx.doi.org/10.1590/S0102-311X2004000200027.

11. Kwon BE, Kim GY, Son YJ, Roh SY, You MA. Quality of life of women with urinary incontinence: a systematic literature review. International Neurourology Journal [Internet], 2010 [cited 2020 may 14]; 14(3):133-8. DOI: https://doi.org/10.5213/inj.2010.14.3.133.

12. Lopes MHBM, Costa JN, Bicalho MB, Casale TE, Camisao AR, Fernandes MLV. Profi le and quality of life of women in pelvic $\mathrm{fl}$ oor rehabilitation. Rev. Bras. Enferm. [Internet], 2018 [cited 2020 Jun 05]; 71(5):2496-505. DOI: http://dx.doi.org/10.1590/00347167-2017-0602.

13. Tamanini JTN, D'Ancona CAL, Botega NJ, Rodrigues NJN. Validation of the Portuguese version of the King's Health Questionnaire for urinary incontinent women. Rev. Saúde Pública [Internet], 2003 [cited 2020 may 14]; 37(2):203-11. DOI: http://dx.doi.org/10.1590/S0034-89102003000200007.

14. Tamanini JTN, Dambros M, D'Ancona CAL, Palma PCR, Rodrigues NJN. Validation of the "International Consultation on Incontinence Questionnaire - Short Form" (ICIQ-SF) for Portuguese. Rev. Saúde Pública [Internet], 2004 [cited 2020 Sep 09]; 38(3):438-44. DOI: https://doi.org/10.1590/S0034-89102004000300015.

15. Souza CCC, Rodrigues AM, Ferreira CE, Fonseca ESM, Bella ZIJD, Girão MJBC, Sartori MGF, Castro RA. Portuguese validation of the Urinary Incontinence-Specific Quality-of-Life Instrument: I-QOL. International Urogynecology Journal [Internet], 2009 [cited 2020 Sep 09]; 20(10):1183-9. DOI: http://dx.doi.org/10.1007/s00192-009-0916-8.

16. Ercole FF, Melo LS, Alcoforado CLGC. Integrative review versus systematic review [editorial]. Reme: Revista Mineira de Enfermagem. 2014; 18(1):9-11. DOI: http://dx.doi.org/10.5935/1415-2762.20140001.

17. Souza MT; Silva MD; Carvalho R. Integrative review: what is it? How to do it?. Einstein [Internet], 2010 [cited 2020 may 20]; 8(1):102-6. DOI: https://doi.org/10.1590/s1679-45082010rw1134.

18. Pintos-Díaz MZ, Alonso-Blanco C, Parás-Bravo P, Fernández-de-las-Peñas $C$, Paz-Zulueta $M$, Fradejas-Sastre $V$, Palacios-Ceña D. Living with Urinary Incontinence: Potential Risks of Women's Health? A Qualitative Study on the Perspectives of Female Patients Seeking Care for the First Time in a Specialized Center. International journal of environmental research and public health [Internet], 2019 [cited 2020 Jun 05]; 16(19):3781-96. DOI: http://dx.doi.org/10.3390/ijerph16193781.

19. Alencar-Cruz JM, Lira-Lisboa Lde. Impact of urinary incontinence on quality of life and its relationship with symptoms of depression and anxiety in women. Rev. Salud Pública [Internet], 2019 [cited 2020 Jun 05]; 21(4):1-8. DOI: https://doi.org/10.15446/rsap.V21n4.50016.

20. Murukesu RR, Singh DKA, Shahar S. Urinary incontinence among urban and rural Community dwellig older women: prevalence, risk factors and quality of life. BMC Public Health [Internet], 2019 [cited 2020 Jun 05]; 19(4):1-11. DOI: http://dx.doi.org/10.1186/s12889-019-6870-6.

21. Padilha JF, da Silva AC, Mazo GZ, Marques CMG. Investigação da qualidade de vida de mulheres com incontinência urinária. Arq. Ciênc. Saúde UNIPAR [Internet], 2018 [cited 2020 Jun 05]; 22(1):43-8. DOI: https://doi.org/10.25110/arqsaude.v22i1.2018.6302.

22. Rett MT, Wardini EB, De Santana JM, Mendonça ACR, Alves AT, Saleme CS. Female urinary incontinence: quality of life comparison on reproductive age and postmenopausal period. Fisioter Mov. [Internet], 2016 [cited 2020 Jun 05]; 29(1):71-8. DOI: https://doi.org/10.1590/0103-5150.029.001.AO07.

23. Lopes MHBM, Higa R. Urinary incontinence restrictions in women's life. Rev. esc. enferm. USP [Internet], 2006 [cited 2020 Sep 9]; 40(1):34-41. DOI: https://doi.org/10.1590/S0080-62342006000100005.

24. Silva NMA, Miranda LM, Machado SRSL, Souza GAS, Mendes IS. O impacto da incontinência urinária feminina na qualidade de vida. In: Anais do $20^{\circ}$ Encontro Latino Americano de Iniciação Científica, $16^{\circ}$ Encontro Latino Americano de Pós-Graduação e $6^{\circ}$ Encontro de Iniciação à Docência; 2016 out 27-28, São José dos Campos, Brasil. São José dos Campos (SP): Educação e Ciência para a Cidadania Global; 2016. p. 1-6. 
25. Faria CA, Moraes JR, Monnerat BR, Verediano KA, Hawerroth PA, Fonseca SC. Effect of the type of urinary incontinence on the quality of life of patients in the public healthcare system in Southeastern Brazil. Rev. Bras. Ginecol. Obstet. [Internet], 2015 [cited 2020 Jun 05]; 37(8):374-80. DOI: http://dx.doi.org/10.1590/so100-720320150005394.

26. Pereira MG, Brittany L, Hall-Faul M, Pedras S. Quality of life of women with urinary incontinence in rehabilitation treatment. Journal of Health Psychology [Internet], 2016 [cited 2020 Jun 05]; 24 (2): 254-263. DOI: http://dx.doi.org/10.1177/1359105316650615.

27. Saboia DM, Firmiano MLV, Bezerra KC, Vasconcelos Neto JA, Oriá MOB, Vasconcelos CTM. Impact of urinary incontinence types on women's quality of life. Rev. Esc. Enferm. USP [Internet], 2017 [cited 2020 Jun 05]; 51:e03266. DOI: http://dx.doi.org/10.1590/S1980-220X2016032603266.

28. Fernandes S, Coutinho EM, Duarte JC, Nelas PAB, Chaves CMCB, Amaral O. Quality of life in women with Urinary Incontinence. Revista de Enfermagem Referência [Internet], 2015 [cited 2020 Jun 05]; 5:93-9. DOI: http://dx.doi.org/10.12707/RIV14042.

29. Göral Türkcü S, Kukulu K. Urinary incontinence and quality of life of women living in nursing homes in the Mediterranean region of Turkey. PSYCHOGERIATRICS [Internet], 2017 [cited 2020 Jun 05]; 17(6):446-52. DOI: https://doi.org/10.1111/psyg.12271. 